\title{
ON THE STIMULATION OF THE DE-EXCITATION OF NUCLEAR ISOMERS IN PLASMA OF A HIGH-CURRENT ELECTRIC DISCHARGE
}

\author{
V. Koltsov
}

\author{
JSC Khlopin Radium Institute, Saint-Petersburg, Russia \\ vladimir-koltsov@yandex.ru
}

\begin{abstract}
The prospects of stimulated de-excitation of nuclear isomers (SDENI) with a trigger transition energy $\Delta E$ up to $\sim 1 \mathrm{keV}$ in a plasma of a high-current electric discharge (HCED) with an electron temperature $\theta \sim \Delta E$ are discussed. An estimate of the probability of the SDENI process in plasma by the mechanism of nuclear excitation by electron capture (NEEC) is obtained. The most promising for the SDENI study are isomers ${ }^{229 m} \mathrm{Th}(\Delta E \approx 8 \mathrm{eV}),{ }^{235 m} \mathrm{U}(\Delta E=76 \mathrm{eV})$, ${ }^{110 m} \mathrm{Ag}(\Delta E=1128 \mathrm{eV})$. To create an energy source most promising isomer is ${ }^{186 m} \operatorname{Re}(\Delta E$ is unknown) with a half-life of $2 \times 10^{5} y$, for which stimulated de-excitation in the laser plasma had been already observed at $\theta \sim 1 \mathrm{keV}$.
\end{abstract}

Keywords: De-excitation of nuclear isomers, Laser plasma, High-current electric discharge plasma.

\section{Introduction}

For more than 60 years, but so far unsuccessfully, attempts have been made to create a controllable source of $\gamma$-radiation or an energy source based on stimulated de-excitation of long-lived nuclear isomers (hereinafter referred to as SDENI). Here, only a brief overview of these studies is given, which is necessary for assessing the prospects for studying the SDENI using the plasma of a high-current electric discharge (hereinafter referred to as the HCED plasma). In an isomeric energy source, the stimulation of de-excitation of a large number of isomers should be either an avalanche or due to an external action of high efficiency. Initially, the SDENI process was supposed to be carried out in the form of a $\gamma$-laser, in which the direct isomeric transitions in nuclei are stimulated by resonant $\gamma$-quanta emitted in the isomeric transitions in other nuclei of the same kind [1-3]. Numerous studies were carried out, but fifty years after the appearance of the idea of a $\gamma$-laser, L. Rivlin, one of the authors of this idea, stated that in all experiments with long-lived isomers the SDENI effect was not observed [4]. The reason for the lack of this effect is the difficulty of creating the resonance conditions for stimulating the radiation for long-lived isomers (see, e.g., [5]). Another reason is that long-lived nuclear isomers spontaneously decay mainly due to internal electron conversion (it is assumed that these are not bare isomeric nuclei, but they have an electronic shell), but, as shown in $[6,7]$, the conversion transition can also be resonantly stimulated by photons, as well as a transition with emission of a $\gamma$-quantum. Therefore, in attempts to resonantly stimulate $\gamma$-ray emission in isomeric transition of longlived isomers by photons, conversion transitions would be stimulated first of all. As far as the author knows, the conversion transitions stimulated by resonance $\gamma$-quanta have not been specifically sought in experiments. However, the possibility of such stimulation corresponds to the experimentally observed decrease in the probability $P_{\text {conv }}$ of spontaneous conversion transitions for ${ }^{235} \mathrm{U}$ (transition of energy $\Delta E=76 \mathrm{eV}$ ), ${ }^{154} \mathrm{Eu}(\Delta E=910 \mathrm{eV}),{ }^{99} \mathrm{Tc}(\Delta E=2173 \mathrm{eV})$ nuclei which are embedding in the metal (see [8] and references therein). This effect is different from changing $P_{\text {conv }}$ due to deformation of the electron shell of atoms or due to the interaction of conversion electrons with the environment. But on the other hand, the effect corresponds to the idea [6, 7] that, just like transitions with $\gamma$-quantum emission, spontaneous conversion transitions are resonantly stimulated by zero-point oscillations of the electromagnetic field, the intensity of which is decreasing inside metal [9].

For the implementation of SDENI also tried to excite the isomers to the higher-lying trigger levels, from where the nucleus can decay into the ground state (see, e.g., reviews [10, 11]. Transitions to trigger levels have a lower multipolarity than direct isomeric transitions and such transitions are easier to induce. For known isomers the energy of trigger transitions is $\Delta E>1 \mathrm{keV}$. Attempts were made for nuclear excitation by electron transition (NEET) in the excited electron shell of atoms, as well as via absorption of photons of resonant energy $\Delta E$ by nuclei, but these methods were not very effective. De-excitation of isomers was observed when they were irradiated with high-energy photons. Thus, the SDENI effect was observed on the ${ }^{99 m}$ Tc isomer $\left(T_{1 / 2}=6 \mathrm{~h}\right)$ under the action of bremsstrahlung radiation with an energy of up to $15 \mathrm{MeV}$ [12] and on the natural isomer ${ }^{180 m} \mathrm{Ta}$ (excitation energy of the isomer $75 \mathrm{keV}$ ) under the action of bremsstrahlung radiation with an energy of up to $6 \mathrm{MeV}$ due to the excitation of trigger states with an energy of about $2 \mathrm{MeV}$ [13]. However, the efficiency of the SDENI process upon irradiation of isomers with high-energy photons is very low, and this SDENI method is not suitable for practical use. When 
isomers were irradiated with photons of lower energies, the SDENI effect was not observed. For example, such studies were widely carried out with the ${ }^{178 m} \mathrm{Hf}$ isomer $\left(T_{1 / 2}=31 \mathrm{y}\right)$, the stimulated de-excitation of which under soft $X$-ray irradiation was announced in [14-16], but more careful experiments (see [17] and references there) and more thorough theoretical analysis [18] did not confirm the observation of the SDENI effect on the ${ }^{178 m 2} \mathrm{Hf}$ isomer. The absence of a strong SDENI effect upon irradiation of isomeric nuclei with low-energy photons is caused by the low efficiency of this method for exciting trigger levels, as well as by the fact that there were no isomers with trigger transitions that can be effectively excited by such photons - trigger transitions of low multipolarity and low energy are needed. Such convenient isomers are currently unknown, but it cannot be argued that such isomers do not exist at all. The point is that it is impossible to accurately determine the parameters of nuclear levels only by calculation, and only those energy levels that are populated in known nuclear reactions are well known. In different nuclear reactions the same nuclei are formed in different excited states, and during the decay of these states in cascades of electromagnetic transitions, different energy levels of these nuclei can appear. As for the excitation of isomers, this is a poorly studied type of nuclear reactions, and new nuclear levels may appear in such reactions, including new trigger levels convenient for de-excitation of isomers. This is especially true for deformed odd-odd nuclei with a complex level structure, for example, the ${ }^{186}$ Re nucleus discussed below. For some isomers, experiments are complicated by the difficulty of obtaining them in sufficiently large quantities. For instance, the ${ }^{178 m 2} \mathrm{Hf}$ isomer was produced only in small amounts of $\sim 10^{15}$ nuclei in accelerators, since usually the most efficient method for producing radionuclides in a reactor is not suitable for the ${ }^{178 m 2} \mathrm{Hf}$ isomer, the neutron cross section for the formation of an isomer from natural hafnium in a reactor is very small - about 2 microbarn $[19,20]$. In addition, it is difficult to prepare targets with a high enrichment in the ${ }^{178 m 2} \mathrm{Hf}$ isomer, since radiochemistry cannot separate the isomeric ${ }^{178} \mathrm{Hf}$ nuclei from the nuclei in ground state that are predominantly formed in nuclear reactions.

At present, the most promising is the search for the SDENI process for isomers in a high-density plasma with an electron temperature of the order of the trigger transition energy $\theta \sim \Delta E$ (hereinafter, the temperature $\theta$ in energy units). Such plasma simultaneously contains intense $X$-ray radiation, intense fluxes of electrons and ions, and a high degree of ionization of atoms with isomeric nuclei. In plasma, the most efficient mechanism of the SDENI is the nuclear excitation by electron capture (NEEC), proposed in 1976 by Goldanskii and Namiot [21] for transitions of ${ }^{235} \mathrm{U}$ nuclei to the first excited state. Subsequently, the NEEC mechanism was investigated in many works (see, e.g., [21-23] and references therein). Recently, in the experiment [24], the NEEC process was observed during deceleration in a metal of fast ions with isomeric nuclei ${ }^{93 m} \mathrm{Mo}$ (the trigger transition energy $\Delta E=4.8 \mathrm{keV}$ ). In the NEEC process a free electron of the plasma is captured by an ionized atom with an isomeric nucleus, the excess energy of the electron goes not only to the emission of a photon or Auger electron, but with the probability of $P_{\text {NEEC }}$ can go to excite the nucleus. NEEC is the inverse process with respect to direct internal electronic nuclear conversion and occurs with the participation of a virtual photon. According to [25], in order of magnitude $P_{\text {NEEC }}$ can be estimated from the formula

$$
P_{\mathrm{NEEC}} \sim \lambda_{\mathrm{res}}^{2} \tau n_{E, \mathrm{res}} \Gamma
$$

where $n_{E \text {,res }}$ is the energy density of electronic states at the electrons energy $E_{\text {res }}$ resonant with the nuclear trigger transition: $E_{\mathrm{res}}=m_{e} v_{e, \mathrm{res}}^{1} / 2=\Delta E-J ; m_{e}$ and $\mathrm{v}_{\mathrm{e}, \mathrm{res}}$ are the mass and the velocity of the plasma electrons; $J$ is the ionization potential of the atomic level at which the plasma election is captured; $\tau$ is the plasma lifetime; $\lambda_{\text {res }}=\hbar / m_{e} v_{\text {e,res }}$ is the de Broglie wavelength; $\hbar$ - Planck's constant divided by $2 \pi$; $\Gamma$ is the width of the conversion transition from the excited nuclear level to the ground state of the nucleus, associated with the half-life $T_{1 / 2}$ of the excited level by the ratio $\Gamma \sim \hbar / T_{1 / 2}$. Formula (1) takes into account the maximum cross section of the NEEC process, which is equal at exact resonance, and the integration of the cross section over the electron energy is approximately represented as the product of the cross section $\sigma_{\text {res }}$ and the flux of plasma electrons with energy near the resonance within the energy width of the transition. Formula (1) was obtained for the transition of the nucleus to the first excited state. If we are talking about the excitation of an isomeric nucleus to a trigger level, which can decay not only back to the isomer, but also via other decay channels, then the width of the trigger level $\Gamma_{\text {trigg }}>\Gamma$, where the width $\Gamma$ is still determined by the probability of transition from the trigger level to the isomer. In this case, the maximum cross section of the NEEC process turns out to be $\Gamma_{\text {trigg }} / \Gamma$ times less than in formula (1) (see, e.g., [23, 26]). However, in this case, the energy range in which the plasma electrons are resonant for the NEEC process increases by the same factor. Thus, the probability of the NEEC process for the excitation of the isomer to the trigger level can still be estimated by formula (1).

Until now, the SDENI process has been mainly studied in laser plasma. In these experiments, a light pulse is directed to a target - a backing with isomeric nuclei deposited on it, as a result a part of the target is converted into plasma. Stimulation of nuclear transitions of energy of tens $\mathrm{keV}$ is possible in a plasma with a temperature of $\theta \sim 10 \mathrm{keV}$, created by laser pulses with a duration of about 1 ps and an energy of tens 
joules, focused on a target into a spot with a diameter of about $100 \mu \mathrm{m}$, with an intensity of more than $10^{18} \mathrm{~W} \mathrm{~cm}^{-2}$. In such plasma $X$-rays of a temperature of tens $\mathrm{keV}$ and the intense fluxes of electrons and protons of energies up to $10 \mathrm{MeV}$ are excited, and there can be nuclear reactions such as $(\gamma, \mathrm{n}),(\mathrm{p}, \mathrm{n})$ ( (see, e.g., review [27]), and the excitation of nuclear isomers, for example, the ${ }^{1034 m} \mathrm{Rh}$ isomer $(\Delta E=39 \mathrm{keV})[28,29]$ or the ${ }^{181 m}$ Ta isomer $(\Delta E=6 \mathrm{keV})[27,30]$. In such plasma the SDENI process was studied for the ${ }^{110 m} \mathrm{Ag}$ isomers $\left(T_{1 / 2}=250 \mathrm{~d}\right)$ [31] (see Section 3.1). For nuclear transitions of energy no more than a few $\mathrm{keV}$, laser pulses of lower intensity but longer duration and grater energy, which form plasma with a temperature of $\theta \sim 1 \mathrm{keV}$, are more efficient, in this case the plasma lifetime $\tau$ can increase up to nanoseconds. In such plasma, the SDENI effect was observed on the ${ }^{186 m}$ Re isomers [32] (see Section 3.2). In contrast to experiments with irradiation of isomers with photons, in plasma experiments it is necessary to have not only a sufficient number of isomeric nuclei, but also a high concentration of the isomer in the target, since the plasma mass is limited.

In experiments with laser plasma, the SDENI effect was observed to be very weak, primarily due to the short plasma lifetime, which is determined only by the duration of the laser pulse and the free expansion velocity of the plasma. It is desirable to increase the plasma lifetime, and here an alternative can be the plasma of a high-current electric discharge (HCED), in particular, the plasma of the electric explosion of conductors [33], for which the lifetime increases due to its magnetic confinement. In the present work, we discuss the prospects of using the HCED plasma for studying the SDENI process.

\section{Probability of the SDENI process in a plasma of a high-current electric discharge (HCED)}

The most powerful HCED facilities, such as the "Angara-5" facility at the Troitsk Institute for Innovation and Thermonuclear Research (TRINITI) (in Moscow, Russia) with a maximum discharge current $I_{\max }=6 \mathrm{MA}$ and a pulse duration up to $\tau=$ 150 ns [34] or the "Z-machine" of Sandia National Laboratories (in Albuquerque, USA) with $I_{\max }=26 \mathrm{MA}$, $\tau=100 \mathrm{~ns}$ [35] make it possible to obtain an HCED plasma with a temperature of $\theta \sim 1 \mathrm{keV}$. This temperature is lower than the record temperatures in the laser plasma, and it can be expected that the temperature of the HCED plasma will be lower than the energy of nuclear trigger transitions $\Delta E$. In this case, the dependence of the probability $P_{\text {SDENI }}$ of the SDENI process on the plasma temperature becomes very important. The use of modern computer codes describing the plasma parameters, in principle makes it possible to calculate with high accuracy the $P_{\text {SDENI }}$ depending on the plasma parameters (see, e.g., [36]).
But for many cases such calculations have not yet been made, and here we will give only a rough estimate of the $P_{\text {SDENI }}$. We will assume that the HCED plasma is close to equilibrium [37], the distribution of $n_{E}$ electrons over their energy $E$ is described by the Maxwell-Boltzmann distribution, and the probability $P_{J}$ of ionization of a level with an ionization potential $J$ in an atom with an isomeric nucleus is described by the Saha formula under the assumption of one-step ionization of this level (see, e.g., [38], page 94):

$$
\begin{gathered}
n_{E}=\frac{2}{\sqrt{\pi}} \frac{n \sqrt{E}}{\theta^{3 / 2}} e^{-E / \theta}, \\
P_{J}=\frac{2 g_{i}}{g_{a}}\left(\frac{m_{e}}{2 \pi \hbar^{2}}\right)^{3 / 2} \frac{\theta^{3 / 2}}{n} e^{-J / \theta} .
\end{gathered}
$$

Here $n$ - is the concentration of electrons in the plasma, $g_{\mathrm{i}} \approx g_{\mathrm{a}}$ - are the statistical weights of states of an ion and a neutral atom with an isomeric nucleus. From formulas (1), (2), we obtain the following simple estimate for the probability $P_{\mathrm{SDENI}}$ of de-excitation of the isomeric nucleus

$$
P_{\mathrm{SDENI}}=P_{\mathrm{NEEC}} P_{J} \sim \frac{1}{\pi^{2}} \frac{\Gamma \tau}{\hbar} e^{-\Delta E / \theta} .
$$

In the approximation used, the probability $P_{\mathrm{SDENI}}$ is proportional to the plasma lifetime $\tau$ and does not depend on the electron concentration. For the highest efficiency of the SDENI process, $\theta \approx \Delta E$ is necessary, although the process is possible at a much lower plasma temperature. This estimate describes well the result of the experiment [39] on the excitation of the ${ }^{236 m} \mathrm{U}$ isomer $(\Delta E=76 \mathrm{eV})$. The ${ }^{235 m} \mathrm{U}$ isomeric transition occurs totally via electronic conversion and the half-life of the isomer $T_{1 / 2}=26 \mathrm{~min}$ corresponds to the width of the isomeric transition $\Gamma \sim \hbar / T_{1 / 2}$. This experiment was carried out at the "Triton" facility at the Troitsk Institute for Innovative Thermonuclear Research (TRINITI). A plasma with a temperature of $\theta \approx 20 \mathrm{eV}$, containing ${ }^{235} \mathrm{U}$ ions, was created by irradiating ${ }^{235} \mathrm{U}$ oxide with $500 \mathrm{keV}$ electrons with a $150 \mathrm{kA}$ current pulse of $30 \mathrm{~ns}$ duration. The probability of excitation of the ${ }^{235 m} \mathrm{U}$ isomer in such a plasma was $P_{\text {SDENI }} \sim 10^{-14}$, which is close to the calculated value by formula (3).

\section{Detecting the SDENI process in pulsed plasma}

It is convenient to consider the method of detecting the SDENI process in pulsed plasma using the experimentally studied isomers ${ }^{110 m} \mathrm{Ag}\left(T_{1 / 2}=250 \mathrm{~d}\right)$ and ${ }^{186 m} \operatorname{Re}\left(T_{1 / 2}=2 \times 10^{5} \mathrm{y}\right)$ (Figures 1,2$)$. The SDENI process can be detected by the emission of "prompt" $\gamma$-quanta directly upon de-excitation of the isomer. In view of the intense $X$-ray radiation of the plasma, these "prompt" $\gamma$-quanta can be detected with high sensitivity only for those isomers for which during the de-exciting the intermediate levels with a half-life $T_{1 / 2}$ longer than the plasma lifetime are populating. 
If the plasma lifetime is less than $T_{1 / 2}=36 \mathrm{~ns}$ for the $118 \mathrm{keV}$ trigger level in the ${ }^{110 m} \mathrm{Ag}$ isomer or $T_{1 / 2}=25 \mathrm{~ns}$ for the intermediate $99 \mathrm{keV}$ level in the ${ }^{186 m}$ Re isomer, then upon detection of "prompt" $\gamma$ quanta it is possible to detune from the plasma $X$-ray background in time. Also, the SDENI process can be detected by "delayed" $\gamma$-quanta emitted in the decay of an intermediate long-lived states of the nucleus, which are formed after de-excitation of the isomers. For the ${ }^{110 m} \mathrm{Ag}$ and the ${ }^{186 m}$ Re isomers, the longlived intermediate states are the ground states of the nuclei. It is just by the time variation of the intensity of "delayed" $\gamma$-quanta after a laser shots to their equilibrium value in experiments [31, 32], the SDENI process was detected on the ${ }^{110 m} \mathrm{Ag}$ and ${ }^{186 m}$ Re isomers. The intensity of "delayed" $\gamma$-quanta can be measured in low-background conditions, however analysis [40] shows that the sensitivity to the SDENI process can be much higher when the process is detected by "prompt" quanta due to their higher intensity. So, when detecting the SDENI for the ${ }^{110 m} \mathrm{Ag}$ isomer by a mediumsized $\gamma$-detector located at a distance of $\sim 10 \mathrm{~cm}$ from the plasma bunch, the minimum detectable probability

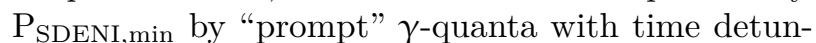
ing from the plasma background $X$-ray radiation is determined only by the number of isomeric nuclei in the plasma and the efficiency of registration of their $\gamma$-quanta and is equal to $\mathrm{P}_{\mathrm{SDENI}}$ min - promt $\sim 10^{-12}$. And $P_{\text {SDENI,min - delay }} \sim 10^{-4}$ when the SDENI process is detecting by "delayed" $\gamma$-quanta. The very large value of $P_{\mathrm{SDENI}}$ min - delay is due to the difficulty of separating $\gamma$-quanta with energies of about $657 \mathrm{keV}$ in decay from the ground and from the isomeric states of the ${ }^{110} \mathrm{Ag}$ nucleus. For the detection of the SDENI process on the ${ }^{186 m}$ Re isomer, respectively, the $\mathrm{P}_{\text {SDENI,min - promt }} \sim 10^{-12}$ and $\mathrm{P}_{\text {SDENI,min - delay }} \sim 10^{-8}$.

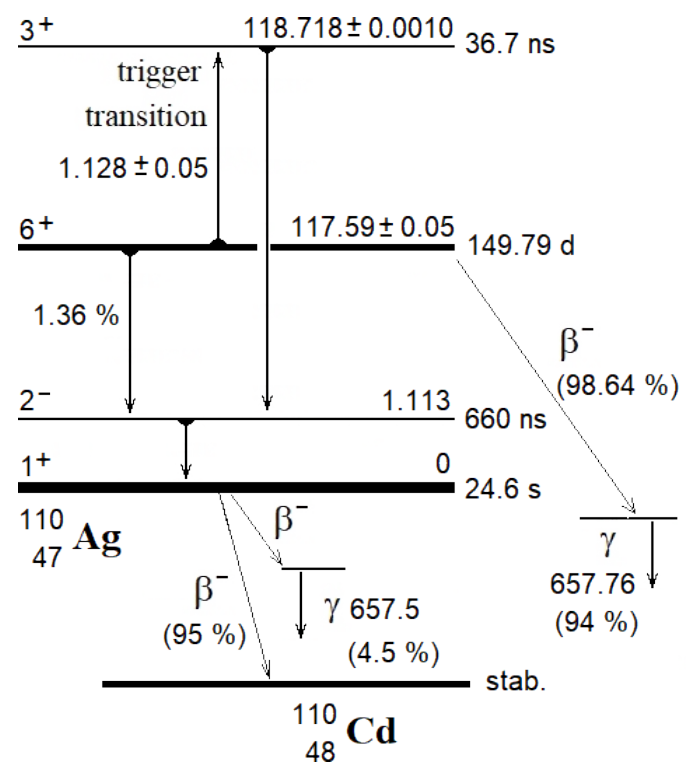

Figure 1. Diagram of the energy levels of the ${ }^{110} \mathrm{Ag}$ nucleus [41]. Levels energies are in $\mathrm{keV}$ $\underline{4-}$ 173.930

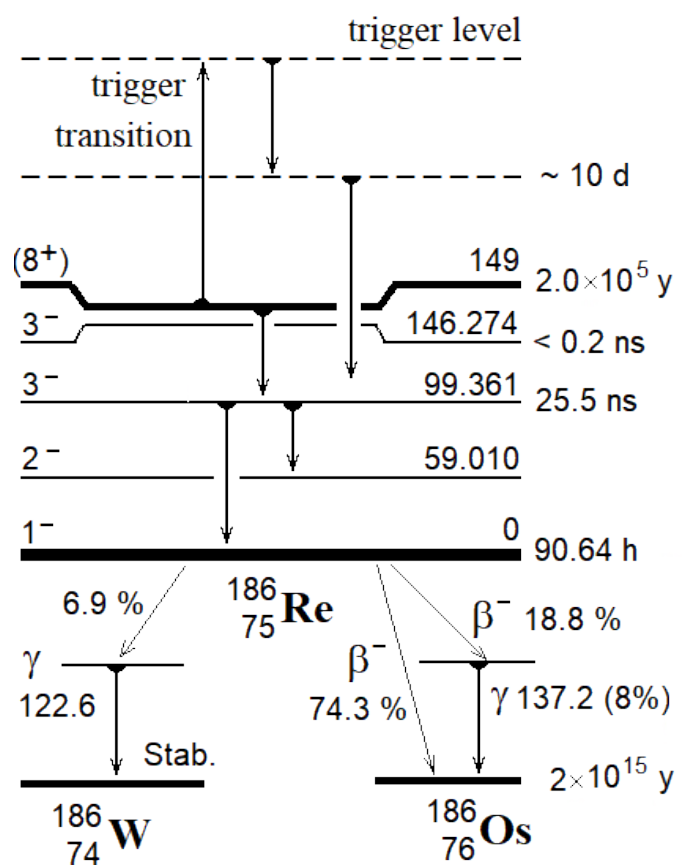

Figure 2. Diagram of the energy levels of the ${ }^{186} \mathrm{Re}$ nucleus [42], the energies are in $\mathrm{keV}$. The dotted lines denotes the trigger level through which the de-excitation of the ${ }^{186 m}$ Re isomer $\left(T_{1 / 2}=2 \times 10^{5} y\right)$ is assumed in the plasma, and the new ${ }^{186 m}$ Re isomer level $\left(T_{1 / 2} \sim\right.$ $10 d)$ which was introduced in [32] to explain the time dependence of the radiation intensity of $137 \mathrm{keV} \gamma$ quanta from the substance of the ${ }^{186 m}$ Re isomeric target after its stay in plasma. The energies of these dotted levels are unknown. The first known level above the ${ }^{186 m}$ Re isomer is the $173 \mathrm{keV}$ level. To avoid cluttering the figure, the reverse transition from the trigger level to the ${ }^{186 m}$ Re isomer is not shown.

\section{Nuclear isomers for studies in HCED plasma}

At present, the study of the SDENI process in the HCED plasma is possible on nuclear isomers with a trigger transition energy up to $\sim 1 \mathrm{keV}$, comparable to the attainable plasma temperature. Excitation of nuclear levels in plasma can be studied on the ${ }^{235 m} \mathrm{U}$ isomer $(\Delta E=76 \mathrm{eV})$ or on the ${ }^{229 m}$ Th isomer $(\Delta E \approx 8 \mathrm{eV})$, its excitation from the ground state of the nucleus was recently observed in an electrical discharge plasma with a temperature of $\theta \approx 10 \mathrm{eV}$ [43]. However, the ${ }^{110 m} \mathrm{Ag}$ and ${ }^{186 m}$ Re isomers seem to be the most promising for research, since de-excitation of these isomers releases a large energy — on the order of $1 \mathrm{MeV}$, and they may be of interest for practical use. 


\subsection{Prospects for research on the ${ }^{110 \mathrm{~m}} \mathrm{Ag}$ isomer $\left(T_{1 / 2}=250 \mathrm{~d}\right)$}

The ${ }^{110 m} \mathrm{Ag}$ isomer for studying the SDENI process in plasma was proposed in paper [40] due to the low trigger transition energy - only $1128 \pm 50 \mathrm{eV}$ (hereinafter, the error is indicated at the level of one standard deviation). Other advantages of this isomer are its long lifetime and the relative ease of isomer preparation. In large amounts the isomer is produced in nuclear reactors, in small amounts it can be obtained at a cyclotron, for example, in the $(\mathrm{p}, \mathrm{n})$ reaction on ${ }^{110} \mathrm{Pd}$ and subsequent separation of silver from palladium on an ion-exchange column [40]. The disadvantage of this isomer is the large multipolarity of the trigger M3 transition, which leads to a low probability of de-excitation of the isomer in plasma.

The specific features of the search technique for the SDENI process on the ${ }^{110 m} \mathrm{Ag}$ isomer were studied experimentally [31] using the laser plasma of the "Progress" facility of the Scientific Research Institute of Optical-Electronic Instruments (Sosnovy Bor, LO, Russia). In these experiments, the laser pulse duration was $1.5 \mathrm{ps}$, the pulse energy was about $20 \mathrm{~J}$, and the intensity varied from $10^{16}$ to $10^{18} \mathrm{~W} \mathrm{~cm}^{-2}$. In laser targets, the ${ }^{110 m} \mathrm{Ag}$ isomer was deposited onto backings of three different metals - lead, platinum, and tungsten. A semiconductor $\gamma$-detector with an energy resolution of $1.8 \mathrm{keV}$ for $650 \mathrm{keV} \gamma$-quanta was located at $10 \mathrm{~cm}$ from the targets. During laser shots, part of the target material converted into plasma with a temperature $\theta$ up to $10 \mathrm{keV}$ and then was deposited on the surface of structural elements of the target chamber near the $\gamma$-detector. One second after the laser shots, the measurement of the $657 \mathrm{keV} \gamma$-quanta intensity began. The idea of this experiment was that upon de-excitation of ${ }^{110 m} \mathrm{Ag}$ isomers in plasma, the population of the ground state of nuclei increases abruptly, which after a laser shot should lead to an exponential decay with a period of $T_{1 / 2}=25 \mathrm{~s}$ of the intensity $I_{657}$ of $657 \mathrm{keV} \gamma$-quanta emitted in the $\beta$ decay of the ground state of ${ }^{110} \mathrm{Ag}$ nuclei with the highest intensity. In the experiment, such a decrease in the $I_{657}$ intensity was indeed observed, but only for targets on platinum backings. The cause for this effect still need to be clarified.

When determining the prospects of studies with the ${ }^{110 m} \mathrm{Ag}$ isomer in the HCED plasma, it is necessary to take into account the probability of the SDENI process, obtained from the estimate of the width $\Gamma \approx 4 \times 10^{-24} \mathrm{eV}$ for the transition from the ${ }^{110 m} \mathrm{Ag}$ isomeric level to the trigger level of 118.7 $\mathrm{keV}$. This estimate of the width $\Gamma$ was obtained in paper [40] from the one-particle probability of a trigger $M 3$ transition and the value of the hindrance factor for this transition, obtained from the systematics of hindrance factors for similar transitions in neighboring nuclei. Taking into account this estimated value of the width $\Gamma$, in an HCED plasma with a temperature $\theta=1 \div 10 \mathrm{keV}$ and a lifetime $\tau=100 \mathrm{~ns}$, the probability of the SDENI process on ${ }^{110 m} \mathrm{Ag}$ isomers by the NEEC mechanism, according to estimate $(3)$, is equal to $\mathrm{P}_{\text {SDENI }} \sim 10^{-17}$. This probability is much less the threshold value of the probability $\mathrm{P}_{\mathrm{SDENI}}$, min - promt $\sim 10^{-12}$ for the possible detecting the SDENI effect on the ${ }^{110 m} \mathrm{Ag}$ isomer (see Section 2) and it would seem that the ${ }^{110 m} \mathrm{Ag}$ isomer is not suitable for experiments. However, for such a final conclusion, it is desirable to clarify the value of $\Gamma$, which probably will be possible to do according to the results of the planned experiment on observing the NEEC process during deceleration of fast ions with ${ }^{110 m} \mathrm{Ag}$ isomeric nuclei [44].

It should be taken into account that for ${ }^{110 m} \mathrm{Ag}$ isomeric nuclei in plasma, the probability of $P_{\mathrm{SDENI}}$ can be increased due to resonant irradiation of the plasma by photons with an energy equal to the trigger transition energy. The transition energy is known with an accuracy of $50 \mathrm{eV}$ and the source of resonant photon radiation can be found. Under resonance irradiation of a plasma containing nuclear isomers, the probability of the SDENI process by the NEEC mechanism increases due to an increase in the concentration of resonant electrons in the plasma via ionization by resonant photons of atoms with isomeric nuclei or with nuclei of their isotopes [45]. An increase in the probability of $P_{\text {SDENI }}$ may also occur due to the stimulation by resonant photons of the emission of a virtual photon participating in the NEEC process, similarly to the resonant stimulation of the emission of a virtual photon during a direct nuclear conversion transition discussed in paper [7]. In paper [45], it was proposed to use the radiation of the plasma itself as a source of resonant radiation when chemical elements with energy of characteristic $X$-rays equal to the energy of the trigger transition are introduced into the plasma. Such near-monoenergetic radiation source is very efficient. For example, when plasma with the temperature of $\theta \sim 1 \mathrm{keV}$ is formed by the action of a $0.5 \mathrm{~ns}$ laser pulse on the surface of metallic tungsten, about $0.5 \%$ of the laser pulse energy is converted into the characteristic radiation of tungsten with energy of $2.2 \mathrm{keV} \mathrm{[46].} \mathrm{The} \mathrm{prospect} \mathrm{of} \mathrm{studying} \mathrm{the} \mathrm{effect} \mathrm{of}$ plasma resonance irradiation with ${ }^{110 m} \mathrm{Ag}$ isomers on the SDENI process makes this isomer interesting for experiments.

\subsection{Prospects for research on the ${ }^{186 m} \mathrm{Re}$ isomer $\left(T_{1 / 2}=2 \times 10^{5} \mathrm{y}\right)$}

The SDENI process on the ${ }^{186 m}$ Re isomer in plasma has already been observed in the experiment [32] on the "Iskra-5" laser facility of the Russian Federal Nuclear Centre "Institute of Experimental Physics" (Sarov, Russia). In the experiment [32], the $300 \mathrm{~J}$ laser pulses with duration of $\tau \approx 0.3 \mathrm{~ns}$ hit a targets with the ${ }^{186 m}$ Re isomer, and plasma with the temperature of $\theta \sim 1 \mathrm{keV}$ was formed. The targets consisted of a thin layer of metallic rhenium with a $10^{-3 \%}$ content of the ${ }^{186 m}$ Re isomer deposited on 
backings of metallic tungsten or stainless steel. The SDENI effect was sought from the time variation of the intensity $I_{137}$ of $137 \mathrm{keV} \gamma$-quanta emitted after $\beta$-decay of the ground state of ${ }^{186}$ Re nuclei by substance of the target after its stay in the plasma. It was expected that if the SDENI process takes place in the plasma, the population of the ground state of ${ }^{186}$ Re nuclei in the plasma will increase abruptly and then after the laser shot for these ${ }^{186}$ Re nuclei there should be an exponential decrease of the $I_{137}$ intensity to the equilibrium value with a period of $90 \mathrm{~h}$. In the experiment, the $I_{137}$ intensity began to measure after placing the ampoules with material from the plasma into the low-background $\gamma$-spectrometer a day after the laser shots, and the time dependence of the $I_{137}$ intensity was really found. If this time dependence of the $I_{137}$ intensity was caused by the SDENI process for the ${ }^{186 m}$ Re isomer in plasma, then $\sim 10^{-5} \%$ of the ${ }^{186 m}$ Re isomers were de-excited in the plasma. However, this dependence of $I_{137}$ was not a smoothly decreasing exponential, but had a maximum, which indicated the de-excitation of ${ }^{186 m}$ Re in plasma via a previously unknown isomeric level, the half-life of which is $T_{1 / 2, m 2} \sim 10 \mathrm{~d}$. This hypothetical new isomeric level ${ }^{186 m 2}$ Re is shown by the dotted line in Figure 2.

In experiment [32], a search was also carried out for the de-excitation of the ${ }^{186 m}$ Re isomer under its irradiation with bremsstrahlung photons of energy up to $40 \mathrm{keV}$ and electrons of energy $10 \mathrm{keV}$ with integral fluxes much higher than in the laser plasma of the "Iskra-5" facility. In these experiments without plasma formation, no stimulated de-excitation of ${ }^{186 m}$ Re was found, which indicates the de-excitation of ${ }^{186 m} \mathrm{Re}$ in plasma precisely by nuclear excitation by capture of plasma electrons (NEEC), and for example, not due to the excitation of the trigger level in inelastic scattering of plasma electrons or due to $X$-ray absorption by ${ }^{186 m}$ Re nuclei. Then, for the probability of the SDENI process on ${ }^{186 m}$ Re isomers in plasma, one can use the estimate (3) obtained on the basis of the NEEC mechanism, and this estimate shows that for the conversion transition from the new isomeric level ${ }^{186 m 2}$ Re to the ${ }^{186 m}$ Re isomer the width $\Gamma<\hbar / T_{1 / 2, m 2} \sim 1 \times^{-21} \mathrm{eV}$ is very small to give the observed SDENI probability of the order of $10^{-5} \%$. Thus, the new isomeric level of ${ }^{186 \mathrm{~m} 2}$ Re cannot be a trigger level for de-excitation of the ${ }^{186 m}$ Re isomer, and this means that there is another unknown level in the ${ }^{186}$ Re nucleus, which acts as a trigger one. In Figure 2 this hypothetical trigger level is indicated by a dotted line. The energy of this level is unknown, but for the possibility of its excitation in plasma with the temperature of $\theta \sim 1 \mathrm{keV}$, the energy of the trigger transition must be no more than a few $\mathrm{keV}$. Thus, the scheme of the de-excitation of ${ }^{186 m}$ Re isomer in the experiment [32] remains unclear at present. However, the assumption of the existence of a new isomeric level $186 \mathrm{~m}^{2} \mathrm{Re}$ has received confirmation on the study of the decay curve from the ${ }^{186}$ Re source which was obtained by irradiating a ${ }^{186} \mathrm{~W}$ tungsten target with $15 \mathrm{MeV}$ protons [47]. The deviation of this decay curve from the exponential showed that in $(\mathrm{p}, \mathrm{n})$ reaction on the ${ }^{186} \mathrm{~W}$ nucleus with a probability of $\sim 1 \%$ a new isomer with a half-life of about $10 \mathrm{~d}$ is populated in the ${ }^{186} \mathrm{Re}$ nucleus. By measuring the spectrum of conversion electrons from the source with the thus obtained new isomer, it is possible to estimate the energy of the trigger transition for the ${ }^{186 m}$ Re isomer.

Results of the laser experiment [32] show the promise of studding the SDENI process on the ${ }^{186 m}$ Re isomer in the HCED plasma. Taking into account the increase by two orders of magnitude in the lifetime of the HCED plasma as compared to the laser plasma, it turns out that the probability of de-excitation of the ${ }^{186 m}$ Re isomer in the HCED plasma with a temperature of $\theta \sim 1 \mathrm{keV}$ can reach a value of $P_{\text {SDENI }} \sim 10^{-3} \%$. With a decrease in temperature $\theta$, according to estimate $(3)$, the probability $P_{\text {SDENI }}$ decreases. The minimum value of $\theta$, at which the SDENI effect can still be detected, depends on the currently unknown trigger transition energy $\Delta E$. At $\Delta E \sim 1 \mathrm{keV}$, it can be expected that up to a temperature of $\theta \sim 100 \mathrm{eV}$ the SDENI effect in the discharge plasma will remain measurable, since, according to estimate $(3)$, at this temperature, $P_{\text {SDENI }} \sim 10^{-7}$, which still remains within the sensitivity range measurements of the SDENI effect by non-equilibrium $\gamma$ ray emission from the isomeric substance with ${ }^{186 m}$ Re after its stay in the plasma. An increase in the probability of SDENI due to the irradiation of plasma with isomeric ${ }^{186 m}$ Re nuclei by resonance photons is still impossible, since the trigger transition energy for ${ }^{186 m} \mathrm{Re}$ is unknown.

In a single electric discharge, the probability of deexcitation of the ${ }^{186 m}$ Re isomer will always be very small for practical use, but the process of the stimulated de-excitation of the isomer can be done multiple times if use the pulsed discharges in an atmosphere of rhenium hexafluoride. Rhenium hexafluoride is a highly volatile compound with a boiling point of only $33.7^{\circ} \mathrm{C}$. Rhenium hexafluoride is formed from elements at $125^{\circ} \mathrm{C}$. In plasma, rhenium hexafluoride will decompose into elemental rhenium and fluorine, but after the discharge, all rhenium can again be converted into gaseous hexafluoride and the discharge can be repeated. Using such a periodic discharge in ${ }^{186 m} \mathrm{Re}$ plasma, it is possible to stimulate the de-excitation of most of the isomers in the discharge chamber. The possibility of the efficient SDENI process on the ${ }^{186 m}$ Re isomer opens the way for the creation of energy sources based on this isomer [48]. The very long half-life of the isomer provides its low specific activity during storage. After stimulation of ${ }^{186 m}$ Re de-excitation, the half-life of the ground state of the nucleus of $90 \mathrm{~h}$ provides a high energy release of about $0.4 \mathrm{~kW} \mathrm{~g}^{-1}$, which will make it possible to use ${ }^{186 m}$ Re as an energy source in many technical devices. It is essential that 
${ }^{186 m}$ Re can be obtained as a pure isomeric substance. When ${ }^{186 m}$ Re is produced from natural rhenium in a nuclear reactor, the isomer is formed in $0.3 \%$ of cases of neutron capture by the ${ }^{185}$ Re isotope $[49,50]$. A mixture of rhenium isotopes can be chemically isolated from the irradiated material. From this mixture an isotope with a mass number $\mathrm{A}=186$ can be isolated by the gas-centrifugal separation using gaseous rhenium hexofluoride [49]. This will be an almost pure isomer of ${ }^{186 m} \mathrm{Re}$, since ${ }^{186}$ Re nuclei in the ground state decay rapidly. It is interesting to note that a metal from a pure isomer will in fact be a new state of matter. The cost of obtaining the ${ }^{186 m}$ Re isomer is comparable to the cost of obtaining an equal mass of ${ }^{238} \mathrm{Pu}$, which is widely used in radionuclide energy sources.

\section{Conclusions}

The carried out consideration of various variants of the implementation of the SDENI process shows the promising prospects of studying the SDENI on isomers with a trigger transition energy $\Delta E$ up to $\sim 1 \mathrm{keV}$ in the plasma of a high-current electric discharge (HCED) with an electron temperature $\theta \sim \Delta E$. In particular, these are isomers ${ }^{229 m} \mathrm{Th}(\Delta E=8 \mathrm{eV})$, ${ }^{235 m} \mathrm{U}(\Delta E=76 \mathrm{eV}),{ }^{110 m} \mathrm{Ag}(\Delta E=1128 \mathrm{eV})$. The most promising for creating an energy source is the $186 m$ Re isomer ( $\Delta E$ is unknown) with a decay half-life of $2 \times 10^{5} \mathrm{y}$, for which stimulated de-excitation in laser plasma at temperature $\theta \sim 1 \mathrm{keV}$ has already been observed. Isomer ${ }^{186 m}$ Re can be obtained by irradiation of natural rhenium with neutrons in a nuclear reactor and can be isolated in pure form by gas-centrifugal separation of rhenium isotopes.

\section{Acknowledgments}

The author thanks for cooperation and very useful discussions of the details and prospects of stimulated de-excitation of nuclear isomers Dr. V.V. Vatulin and Dr. N.V. Zhidkov from the Russian Federal Nuclear Center "Institute of Experimental Physics", Dr. G.S. Volkov and Dr. E.V. Grabovsky from the Troitsk Institute for Innovative and Fusion Research, Dr. F.F. Karpeshin from the Mendeleev Institute of Metrology (St. Petersburg), Professor I.A. Mitropolskiy from St. Petersburg State University. The author thanks for the interesting and useful discussions on the reports of this work the participants of the seminars in the above scientific centers, the participants of the seminar of corresponding member of the Russian Academy of Sciences A.A. Vorobyov at the Konstantinov Institute of Nuclear Physics (St. Petersburg) and the seminar of academician V.Ya. Panchenko at the Kurchatov Institute (Moscow), the participants of conferences at which the materials presented in this work were considered. In particular, the author thanks physicists from the Czech Technical University in Prague, who noted the impossibility of a trigger transition to the isomeric level ${ }^{186 m 2}$ Re in stimulated de-excitation of the ${ }^{186 m}$ Re isomer because its long lifetime.

\section{References}

[1] L. A. Rivlin. Su inventor's certificate no. 621256 on jan. 10, 1961. SU Bull. Inventions, 23:220, 1979.

[2] V. Vali and W. Vali. Induced $\gamma$-ray emission. Proc. IEEE, 51:182-184, 1963.

[3] G. C. Baldwin, J. P. Neissel, J. Terhune, and L. Tonks. Trans. Am. Nucl. Soc., 6:178, 1963.

[4] L. A. Rivlin. Nuclear gamma-ray laser: the evolution of the idea. Quantum Electronics, 37(8):723-744, 2007.

[5] G. C. Baldwin and J. C. Solem. Recoilless gamma-ray lasers. Rev. Mod. Phys., 69(4):1085-1117, 1997. doi:10.1103/RevModPhys.69.1085.

[6] V. V. Koltsov. On the probability of the conversion transition of ${ }^{235 m} \mathrm{U}$ nuclei in silver. Bull. Russ. Acad. Sci: Phys., 57:93-99, 1993.

[7] V. V. Koltsov. On resonant properties of nuclear conversion transition and on possibility of its resonant stimulation. Bull. Russ. Acad. Sci: Phys., 83(9):1144-1148, 2019.

[8] V. V. Koltsov, A. A. Rimskii-Korsakov, and V. V. Karasev. Influence of a metallic matrix on the probability of the transition at $910 \mathrm{ev}$ in ${ }^{154} \mathrm{Eu}$ nuclei". Bull. Russ. Acad. Sci: Phys., 80(8):884-887, 2016. doi:10.3103/S1062873816080256.

[9] V. V. Koltsov. Method for describing the influence of the dielectric properties of the medium on the probability of the isomeric transition $e=3.5 \mathrm{eV}$ in the ${ }^{229 m}$ Th nucleus. Bull. Russ. Acad. Sci: Phys., 64(3):447-451, 2000.

[10] J. J. Carroll. An experimental perspective on triggered gamma emission from nuclear isomers. Laser Phys. Lett., 1(6):275-281, 2004. doi:10.1002/lapl.200310065.

[11] S. A. Karamian. Prospects for isomeric energy release. Phys. Part. Nucl., 39(4):490-525, 2008.

[12] I. Bikit, I. V. Anicin, M. Kimar, J. Silvka, M. Veskovic, and L. J. Conkic. Depopulation of the $6 \mathrm{~h}$ isomeric state of ${ }^{99} \mathrm{Tc}$ by photon irradiation. J. Phys. G: Nucl. Part. Phys., 19:1359-1364, 1993. doi : 10.1088/0954-3899/19/9/014.

[13] C. B. Collins, C. D. Eberhard, J. W. Glesener, and J. A. Anderson. Depopulation of the isomeric state ${ }^{180 m}$ Ta by the reaction ${ }^{180 m} \mathrm{Ta}\left(\gamma, \gamma^{\prime}\right)^{180} \mathrm{Ta}$. Phys. Rev., 37:2267-2269, 1988. doi:10.1103/PhysRevC.37.2267.

[14] C. B. Collins, F. Davanloo, M. C. Iosif, R. Dussart, J. M. Hicks, S. A. Karamian, C. A. Ur, I. I. Popescu, V. I. Kirischuk, J. J. Carroll, H. E. Roberts, P. McDaniel, and C. E. Crist. Accelerated emission of gamma rays from the $31 \mathrm{y}$ isomer of ${ }^{178} \mathrm{Hf}$ induced by X-ray irradiation. Phys. Rev. Let., 82(4):695-698, 1999. doi:10.1103/PhysRevLett.82.695.

[15] C. B. Collins, N. C. Zoita, A. C. Rusu, M. C. Iosif, D. T. Camase, F. Davanloo, S. Emura, T. Uruga, R. Dussart, J. M. Pouvesle, C. A. Ur, I. I. Popescu, V. I. Kirischuk, N. V. Strilchuk, and F. J. Agee. Tunable synchrotron radiation used to induce $\gamma$-emission from the 31 y isomer of ${ }^{178} \mathrm{Hf}$. Europhys. Lett., 57(5):677-682, 2002. doi:10.1209/epl/i2002-00516-1. 
[16] C. B. Collins, N. C. Zoita, F. Davanloo, Y. Yoda, T. Uruga, J. M. Pouvesle, and I. I. Popescu. Nuclear resonance spectroscopy of the $31 \mathrm{y}$ isomer of ${ }^{178} \mathrm{Hf}$. Laser Phys. Lett., 2(3):162-167, 2005. doi:10.1002/lapl.200410154.

[17] I. Ahmad, J. C. Banar, J. A. Becker, T. A. Bredeweg, J. R. Cooper, D. S. Gemmell, A. Kraemer, A. Mashayekhi, D. P. McNabb, G. G. Miller, E. F. Moore, P. Palmer, L. N. Pangault, R. S. Rundberg, J. P. Schiffer, S. D. Shastri, T.-F. Wang, and J. B. Wilhelmy. Search for $x$-ray induced decay of the $31 \mathrm{y}$ isomer of ${ }^{178} \mathrm{Hf}$ using synchrotron radiation. Phys. Rev., C71:024311-16, 2005. doi:10.1103/PhysRevC.71.024311.

[18] E. Tkalya. Induced decay of ${ }^{178 m 2}$ Hf: Theoretical analysis of experimental results. Phys. Rev., C71:0246068, 2005. doi:10.1103/PhysRevC.71.024606.

[19] S. A. Karamian, J. J. Carroll, J. Adam, E. N. Kulagin, and E. P. Shabalin. Production of long-lived hafnium isomers in reactor irradiations. High Energy Density Physics, 2:48-56, 2006. doi:10.1016/j.hedp.2006.03.001.

[20] A. Izhutov, A. Petelin, M. Gromov, S. Sazontov, V. Pimenov, V. Afanasiev, and V. Koltsov. Production of the ${ }^{178 m 2} \mathrm{Hf}$ isomer in nuclear reactor. LXX International conference "Nucleus - 2020", 11-17 October 2020. Online part. Book of Abstracts, page 135, 2020.

[21] E. V. Tkalya. Nuclear-excitation in atomic transitions (neet process analysis). Nucl. Phys., A539(2):209-222, 1992. doi : 10.1016/0375-9474(92)90267-N.

[22] F. F. Karpeshin, M. B. Trzhaskovskaya, and J. Zhang. Prospect of triggering the ${ }^{178 m 2} \mathrm{Hf}$ isomer and the role of resonance conversion". Eur. Phys. J., A39:341-348, 2009. doi:10.1140/epja/i2008-10714-3.

[23] W. S. A. Pálffy. Theory of nuclear excitation by electron capture for heavy ions. Phys. Rev., A73:01271513, 2006. doi:10.1103/PhysRevA.73.012715.

[24] C. J. Chiara, J. J. Carroll, M. P. Carpenter, J. P. Greene, D. J. Hartley, R. V. F. Janssens, G. J. Lane, J. C. Marsh, D. A. Matters, M. Polasik, J. Rzadkiewicz, D. Seweryniak, S. Zhu, S. Bottoni, A. B. Hayes, and S. A. Karamian. Isomer depletion as experimental evidence of nuclear excitation by electron capture. Nature, 554:216-218, 2018. doi:10.1038/nature25483.

[25] V. I. Goldanskii and V. A. Namiot. On the excitation of isomeric nuclear levels by laser radiation through invers internal electron conversion". Phys. Lett. B, 62(4):393-394, 1976. doi : 10.1016/0370-2693(76)90665-1.

[26] M. R. Harston and J. F. Chemin. Mechanisms of nuclear excitation in plasmas. Phys. Rev., C59(5):24622473, 1999. doi:10.1103/PhysRevC.59.2462.

[27] A. V. Andreev, V. M. Gordienko, and A. B. Savel'ev. Nuclear processes in a high-temperature plasma produced by an ultrashort laser pulse. Quantum Electron, 31(11):941-956, 2001.

[28] V. I. Afonin, A. G. Kakshin, and A. V. Mazunin. Experimental study of the excitation of rhodium isomer in a plasma produced by a picosecond laser pulse. Plasma Physics Reports, 36(3):250-255, 2001. doi:10.1134/S1063780X10030062.
[29] V. I. Afonin, D. A. Vichliev, A. G. Kakshin, I. I. Kostenko, A. V. Mazunin, and K. V. Safronov. Experimental study of excitation of isomeric states of rodium-103 nuclei at the Sokol-P laser facility. Problems of Atomic Science and Technology. Series Thermonuclear Fusion, 3:75-84, 2012. (in Russian).

[30] O. Renner, L. Juha, J. Krasa, E. Krousky, M. Pfeifer, A.Velyhan, C. Granja, J. Jakubek, V. Linhart, T. Slavicek, Z. Vykydal, S. Pospisil, J. Kravarik, J. Ullschmied, A. Andreev, T. kampfer, I. Uschmann, and E. Forster. Low-energy nuclear transitions in subrelativistic laser-generated plasmas. Laser and Particle Beams, 26:249-257, 2008. doi:10.1017/S0263034608000293.

[31] V. G. Borodin, V. V. Vatulin, N. V. Zhidkov, I. P. Elin, V. M. Komarov, V. Karasev, V. Koltsov, V. Malinov, V. Migel, V. Popikov, A. Rimskii-Korsakov, N. Suslov, and A. Charukhchev. Search for de-excitation of the ${ }^{186 m}$ Re and ${ }^{110 m} \mathrm{Ag}$ nuclear isomers in plasma of high-power picosecond laser pulse. Phys. Atom. Nucl., 82(12):1706-1713, 2019. doi:10.1134/S1063778819120032.

[32] V. V. Vatulin, N. V. Zhidkov, A. A. RimskiiKorsakov, V. V. Karasev, V. V. Koltsov, A. I. Kostylev, and G. V. Tachaev. Searching for stimulated deexcitation of the ${ }^{186 m}$ Re nuclei isomeric state in Iskra-5 laser plasma. Bull. Russ. Acad. Sci: Phys., 81(10):11591162, 2017. doi:10.3103/S1062873817100252.

[33] V. Koltsov. On stimulation of nuclear isomer de-excitation in plasma of electric explosion of conductors. Abstracts of LXVIII meeting on nuclear spectroscopy and nuclear structure "Nucleus - 2018", page 127, July 2-6, 2018, Voronezh, Russia.

[34] Z. A. Albikov, E. P. Velishov, A. N. Veretenikov, V. A. Glukhikh, and E. V. G. et all. Experimental complex Angara-5-1. Soviet Atomic Energy, 68(1):26-35, 1990. (in Russian).

[35] https://www.sandia.gov. arXiv:https://www. sandia.gov.

[36] Y. Wu, J. Gunst, C. Keitel, and A. Palffy. Tailoring laser-generated plasmas for efficient nuclear excitation by electron capture. Phys. Rev. Let., 120:052504, 2018. doi:10.1103/PhysRevLett.120.052504.

[37] V. A. Burtsev, N. V. Kalinin, and A. V. Luchinsky. Electrical explosion of conductors and its use in electrophysical facilities. Energoatomizdat, Moscow, 1990. (in Russian).

[38] A. Fridman. Plasma Chemistry. Cambridge University Press, 2008.

[39] R. V. Arutyunayn, I. Y. Baranov, L. A. Bolshov, V. D. Vikharev, S. A. Dorshakov, V. V. Koltsov, V. A. Kornilo, A. A. Krivolapov, D. D. Malyuta, G. A. Polyakov, A. A. Rimskii-Korsakov, V. P. Smirnov, and E. V. Tkalya. Excitation of the low-lying isomer of uranium-235 in a plasma generated by an electron beam. Preprint IAE-5087/6. Kurchatov Institute of Atomic Energy, Moscow, Russia, 1990. (in Russian).

[40] V. V. Koltsov. The possibility of the triggered de-excitation of the ${ }^{110 m} \mathrm{Ag}$ isomer in laser plasma. Bull. Russ. Acad. Sci: Phys., 80(10):1320-1324, 2018. doi:10.3103/S1062873818100143. 
[41] G. Gurdal and F. G. Kondev. Nuclear data sheets for $\mathrm{A}=110 . N D S, 113: 1315-1561,2012$.

doi:10.1016/j.nds.2012.05.002.

[42] C. M. Baglin. Nuclear data sheets for $\mathrm{A}=186 . N D S$, 99:1-196, 2003.

doi:doi.org/10.1006/ndsh.2003.0007.

[43] P. V. Borisyuk, E. V. Chubunova, N. N. Kolachevsky, Y. Y. Lebedinskii, O. S. Vasiliev, and E. V. Tkalya. Excitation of ${ }^{229} \mathrm{Th}$ nuclei in laser plasma: the energy and half-life of the low-lying isomeric state, 1 Apr. 2018. arXiv: $1804.00299 \mathrm{v} 1$.

[44] L. Syrockiy, K. Słabkowska, M. Polasik, J. R. E. Weder, J. Carroll, and C. Chiara. Studies of optimal conditions for depletion of the ${ }^{110 m} \mathrm{Ag}$ isomer via nuclear excitation by electron capture in a beam-based scenario". Acta Physica Polonica B, 51(1):393-398, 2020. doi:10.5506/APhysPolB .51.393.

[45] V. V. Koltsov. On excitation of nuclear transitions in plasma under its irradiation with resonant photons. Abstracts of LXIX international conference on nuclear spectroscopy and nuclear structure "Nucleus - 2019", 102(3):177, July 1-5, 2019. Dubna, Russia.

[46] V. V. Vatulin, O. Vinokurov, K. A. Volkova, R. V. Garanin, P. D. Gasparyan, G. M. Eliseev, N. V. Zhidkov,
V. P. Kovalenko, P. G. Kuznetsov, M. A. Kulikov, D. I. Martsovenko, L. Z. Morenko, N. P. Pleteneva, N. A. Ryabikina, V. V. Smirnov, N. A. Suslov, G. V. Tachaev, A. V. Tikhonov, and E. S. Tsoi. The study of the generation of line $\mathrm{x}$-ray radiation in laser plasmas of various materials at the Iskra- 5 facility. Problems of Atomic Science and Technology, Series Theoretical and Applied Physics, 3:3-27, 2014 (in Russian).

[47] V. V. Karasev, V. V. Koltsov, and A. A. RimskiiKorsakov. Search for a new isomeric state in the ${ }^{186} \mathrm{Re}$ nucleus". Bull. Russ. Acad. Sci: Phys., 82(10):12371239, 2018. doi:10.3103/S106287381810012X.

[48] V. Koltsov. Prospects for plasma excitation of ${ }^{186 m}$ Re nuclear isomer. LXX International conference "Nucleus 2020", Book of Abstracts., page 190, 11-17 October 2020.

[49] V. Koltsov. Production of the ${ }^{186 m}$ Re isomer in nuclear reactor. LXX International conference "Nucleus - 2020". Book of Abstracts, page 136, 11-17 October 2020. Online part.

[50] D. Seegmiller, M. Linder, and R. Meyer. ${ }^{186}$ Re: Nuclear structure and an isomer of half-live $2 \times 10^{5} \mathrm{y}$. Nucl. Phys., A185:94-112, 1972. doi : 10.1016/0375-9474(72) 90553-2. 